\title{
Abstract: Quantitative Photoakustische Tomografie durch lokale Kontextkodierung
}

\author{
Janek Gröhl, Thomas Kirchner, Lena Maier-Hein
}

Abteilung für Computer-assistierte Medizinische Interventionen, DKFZ Heidelberg

j.groehl@dkfz.de

Photoakustische Tomografie (PAT) ist eine neue strukturelle und funktionale Bildgebung, welche die Darstellung von optischen Absorbern im Gewebe ermöglicht. Im Gegensatz zu anderen weit verbreiteten Modalitäten ermöglicht PAT die Aufnahme von Bildern in Echtzeit und ohne Strahlungsbelastung. Dabei bietet PAT eine hohe räumliche und zeitliche Auflösung. Anders als etablierte optische Bildgebungsverfahren ist PAT in der Lage, optische Parameter mehrere Zentimeter tief im Gewebe zu messen. Jüngste Studien zeigen vielversprechende klinische Anwendungen, unter anderem die Darstellung von Wächterlymphknoten [1] oder die Beobachtung der Aktivität von entzündlichen Prozessen bei Morbus Crohn [2].

Eine der Hauptherausforderungen für die klinische Anwendbarkeit von PAT ist, dass optische Eigenschaften des Gewebes nicht zuverlässig quantifiziert werden können. Dies ist jedoch eine wichtige Voraussetzung für viele multispektrale Anwendungen wie beispielsweise die Schätzung der lokalen Blutsauerstoffsättigung. Das gemessene Signal ist nicht nur proportional zum optischen Absorptionskoeffizienten des Gewebes, sondern zusätzlich abhängig von der optischen Fluenz, welche wiederum von den optischen Eigenschaften des Gewebes und der Art der Beleuchtung abhängig ist. Bisherige Ansätze in der Literatur für die Korrektur für diesen Term scheitern an geringer Robustheit und langen Rechenzeiten.

In diesem Beitrag präsentieren wir einen neuartigen Ansatz zur Quantifizierung von PAT. Mittels einer Methode zur Schätzung der Fluenz in jedem Voxel aus lokaler Kontextinformation kann das PAT Signal korrigiert werden. Erste in silico Experimente zeigen eine hohe Genauigkeit und Robustheit des neuen Konzepts bei um Größenordnungen geringeren Rechenzeiten im Vergleich zu verwandten Verfahren. Sollte eine ähnliche Performanz in vivo erreicht werden, ist das Potential des neuen Ansatzes für die klinische Anwendung enorm.

\section{Literaturverzeichnis}

1. Erpelding TN, Garcia-Uribe A, Krumholz A, et al.; International Society for Optics; Photonics. A dual-modality photoacoustic and ultrasound imaging system for noninvasive sentinel lymph node detection: preliminary clinical results. SPIE BiOS. 2014; p. 894359.

2. Waldner MJ, Knieling F, Egger C, et al. Multispectral optoacoustic tomography in Crohn's disease: non-invasive imaging of disease activity. Gastroenterol. 2016. 